\title{
Impact of desynchronization on PPM UWB systems: a capacity based approach
}

\author{
Mohamed Kamoun \\ Motorola Labs ${ }^{1} /$ CNRS LSS ${ }^{2}$ \\ kamoun@crm.mot.com
}

\author{
Marc de Courville \\ Motorola Labs ${ }^{1}$ \\ courvill@crm.mot.com
}

\author{
Laurent Mazet \\ Motorola Labs ${ }^{1}$ \\ mazet@crm.mot.com
}

\author{
Pierre Duhamel \\ CNRS LSS ${ }^{2}$ \\ Pierre.Duhamel@lss.supelec.fr
}

${ }^{1}$ Centre de Recherche Motorola Paris, Parc Les algorithmes Saint-Aubin 91193 Gif-sur-Yvette France Tel: +33 169357700

Fax: +33169354801

${ }^{2}$ Laboratoire des signaux et systmes (L2S) Suplec - 3 rue Joliot-Curie 91190 Gif-sur-Yvette (France)Tel: +331 69851712

Fax:+33169851769

\section{Abstract}

In this paper the capacity of a specific PPM (Pulse Position Modulation) Ultra Wide Band (UWB) system using a semi-digital demodulator architecture is evaluated. Then the impact of time desynchronization between the local pulses templates and the received signal on the capacity of the link is assessed. The goal being to quantify the jitter effect and delay estimation error on this capacity and issue design recommendations for real system construction.

\section{Introduction}

Ultra wide band (UWB) is a candidate technology for short range and high data rate communications. Its principle is to emit over a huge spectrum band at a very low power avoiding interference with existing systems such as for example existing WLANs (WireLess Area Network) and ISM band devices.

Since according the FCC, an UWB system is defined by its spectrum mask, many physical layers are being proposed to address this type of communication in standardization bodies [1]. There are carrier based technologies like multi band OFDM and Direct Sequence spread spectrum and carrierless systems which consist in the most cases in transmitting very short pulses with a very low duty cycle. Many modulation variants are thus possible: data symbols can be coded with the pulse amplitude (PAM), pulse polarity (POL) or phase and pulse position with respect to known time [6] (PPM). This paper focuses on the PPM modulation.

In this contribution, we evaluate the mutual information between transmitter and receiver with a given modulation/demodulation scheme which relies on a semidigital correlation demodulator. Such evaluation allows the calculation of maximum available data rate.

Capacity analysis for PPM systems has already been studied in optical communication and also in UWB with orthogonal pulses context $[4,13]$. In what follows, since PPM modulation requires a very accurate timing, we propose in the capacity derivation to take into account the desynchronization between the transmitter and the receiver because it can severely restrict the system capac- ity. This evaluation is performed for a single user UWB PPM link in a non-orthogonal pulse context and with a correlation based demodulator architecture. The considered desynchronization captures both the time jitter introduced by the receiver oscillator and also the peak position detection error resulting from channel estimation. Such analysis provides guidance on the time accuracy required in the design of a PPM UWB receivers.

This paper is organized as follows: first a a brief description of the PPM modulation/demodulation is presented in section II. Section III derives the capacity of PPM UWB links with the chosen demodulator architecture. In section IV these calculations are used with a simple desynchronization model to provide practical numerical results. Based on these results, recommendations for the design of PPM UWB systems based on capacity evaluation are proposed.

\section{PPM semi-digital demodulator}

This section intends to settle the notations and also presents the semi-digital demodulator architecture of the PPM UWB system considered.

\section{A PPM signal structure}

Assuming a time division into small slots called chips of duration $T_{c}$, the PPM signal can be modeled as:

$$
s(t)=\sum_{k=-\infty}^{+\infty} a_{\left\lfloor\frac{k}{N_{f}}\right\rfloor} w\left(t-c_{k} T_{c}-f\left(b_{\left\lfloor\frac{k}{N_{f}}\right\rfloor}\right)\right)
$$

where

- $a_{k}, k \in \mathbb{Z}$ are PAM coded symbols. For PPM-only system, $a_{k}=1, \forall k \in \mathbb{Z}$

- $b_{k}, k \in \mathbb{Z}$ are the PPM coded symbols.

- $N_{f}$ is the number of pulses per symbol of data.

- $f$ is the mapping function of PPM symbols. This function corresponds to a small delay relative to the beginning of the time slot. For PAM only system $f=0$ 
- $c_{k}, k \in \mathbb{Z}$ is an increasing random sequence. We assume that the sequence is given by:

$$
c_{k}=k \cdot N_{f}+d_{k}
$$

where $d_{k}$ is a bounded random sequence which values are in the interval $\left[0 . . d_{\max }\right]$

The pseudorandom sequence $c_{k}$ avoids a peak in the signal spectrum density at the frame repetition frequency. Throughout this paper, we consider that all calculations are independent of this sequence.

- $w(t)$ is the second derivative of the original pulse. Indeed, due to the antenna effect on ultra wide band signals [12] the original pulse which is supposed to be gaussian is derived twice and $w(t)$ is defined by the monocycle pulse:

$$
w(t)=\left(1-\frac{2 \pi^{2} t^{2}}{\tau^{2}}\right) e^{-\frac{\pi^{2} t^{2}}{\tau^{2}}}
$$

\section{B PPM correlator demodulator}

The chosen demodulator relies on cross-correlating the received signal by one or more local pulse templates derived by time shift of the original pulse $w(t): w\left(t-\tau_{i}\right)$. These templates are stored in a digital form in the receiver. The correlation is achieved by an analog correlator but the local pulses are generated from their digital storage by the mean of DACs (Digital to Analog Converter) as shown in figure 1 . The correlation needs a very high frequency sampler: $15 \mathrm{GHz}$ for a pulse width of $200 \mathrm{ps}$, its value is evaluated by ADC's only at the pulse rate and not at the sample rate. All the system parameters used here are derived from a system studied in the framework of the European project UCAN [2].

Each correlator provides the correlation between the received signal and one of the local pulses. Increasing the number of correlators allows a better resolution on the arrival time of received pulse.

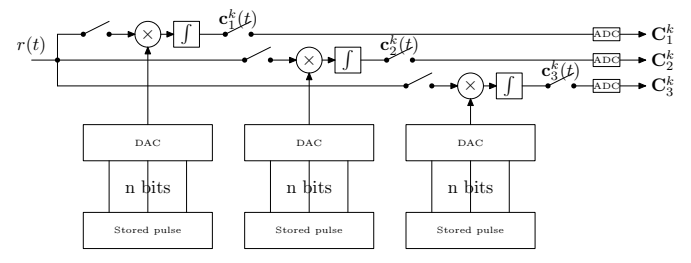

Figure 1: PPM demodulator structure

At the output of the demodulator, the correlations are stacked into vector $\mathbf{C}^{k}:=\left[\mathbf{C}_{0}^{k} \ldots \mathbf{C}_{N-1}^{k}\right]^{T} . \mathbf{C}_{i}^{k}$ represents the correlation between the received signal and the $i$ th local pulse during the $k$ th symbol. For now, it is assumed that local pulses are shifted versions of the original pulse $w(t)$. As these pulses are generated from their local storage in the receiver, the resolution on their shift time is infinite.
With an AWGN channel the received signal is given by:

$$
r(t)=s(t)+\eta(t)
$$

where $\eta(t)$ is Gaussian white noise of variance $\sigma^{2} . \mathbf{C}_{i}^{k}$ can then be retrieved from the autocorrelation function of the pulse by

$$
\begin{aligned}
\mathbf{C}_{i}^{k} & =\int_{-\infty}^{\infty} w\left(t-c_{k} \cdot T_{c}-\tau_{i}\right) r(t) d t \\
& =S_{w}\left(b_{\left\lfloor\frac{k}{N_{f}}\right\rfloor}-\tau_{i}\right)+\|w\| \eta_{i}^{k}
\end{aligned}
$$

where

- $S_{w}$ is the autocorrelation function of $w(t)$

- $\eta_{i}^{k}$ is a sample of a gaussian noise which has the same energy as the original continuous gaussian noise $\eta(t)$. Nevertheless $\eta_{i}^{k}, i \in[0 \ldots N-1]$ can be correlated.

- $\|w\|^{2}$ is the energy of the local pulse templates,

$$
\|w\|^{2}=\int_{-\infty}^{\infty} w(t)^{2} d t
$$

When considering a two correlators demodulator, the optimum choice for $\tau_{i}$ is to align with the original possible pulse positions: $\tau_{i}=f(i)$ (matched filter).

\section{Capacity evaluation of binary PPM links}

\section{A Mutual information of a PPM link}

Only binary PPM systems are considered throughout this paper i.e $N=2$. The PPM mapping function is given by

$$
f(b)=b \Delta
$$

Where $\Delta$ is the time shift between the symbol $b=0$ and symbol $b=1$. The mutual information between the transmitter and the receiver is given by [8]:

$$
I(C, b)=H(C)-H(C \mid b)
$$

The second term in eq. (7) is the entropy of the bidimensional Gaussian noise $\eta^{\mathbf{k}}=\left[\eta_{0}^{k} \eta_{1}^{k}\right]^{T}$. It does not depend on the data distribution $\left(p_{0}, p_{1}\right)$ and is given by:

$$
H(C \mid b)=\frac{1}{2} \log _{2}\left(\operatorname{det}\left(2 \pi e \mathbf{R}_{\eta}\right)\right)
$$

where $\mathbf{R}_{\eta}$ is the covariance matrix of $\eta^{k} . \mathbf{R}_{\eta}$ is given by:

$$
\mathbf{R}_{\eta}=\sigma^{2}\left[\begin{array}{ll}
S_{w}(0) & S_{w}(\Delta) \\
S_{w}(\Delta) & S_{w}(0)
\end{array}\right]
$$


and $\sigma^{2}$ is the variance of the original noise $\eta(t)$. The first term in eq. (7) is the entropy of the received correlation $\mathbf{C}_{k}$

$$
H(C)=\int_{-\infty}^{\infty} \mathcal{P}(\mathbf{C}) \log _{2} \frac{1}{\mathcal{P}(\mathbf{C})} \mathrm{d} \mathbf{C}
$$

where $\mathcal{P}(\mathbf{C})$ is given by:

$$
\mathcal{P}(\mathbf{C})=p_{0} \mathcal{P}(\mathbf{C} \mid b=0)+p_{1} \mathcal{P}(\mathbf{C} \mid b=1)
$$

where $p_{0}=\mathcal{P}(b=0)$ and $p_{1}=\mathcal{P}(b=1)$. The integral in eq. (10) is not easy to calculate, but it can be evaluated numerically.

The capacity of the link corresponds to the pair $\left(p_{0}, p_{1}\right)$ that maximizes $I(\mathbf{C}, b)$. Due to the symmetry between $p_{0}$ and $p_{1}, H(\mathbf{C})$ has an extremum for $p_{0}=p_{1}=\frac{1}{2}$ which is a maximum in our case.

$\mathcal{P}(\mathbf{C})$ can be expanded to

$$
\begin{aligned}
\mathcal{P}(\mathbf{C}) & = \\
& \frac{p_{0} e^{-\left(\mathbf{C}-\mathbf{C}_{\mathbf{0}}\right)^{*} \mathbf{R}_{\eta}{ }^{-1}\left(\mathbf{C}-\mathbf{C}_{\mathbf{0}}\right)}+p_{1} e^{-\left(\mathbf{C}-\mathbf{C}_{\mathbf{1}}\right)^{*} \mathbf{R}_{\eta}{ }^{-1}\left(\mathbf{C}-\mathbf{C}_{\mathbf{1}}\right)}}{\sqrt{\operatorname{det}\left(2 \pi \mathbf{R}_{\eta}\right)}}
\end{aligned}
$$

Where $\mathbf{C}_{\mathbf{0}}$ (respectively $\mathbf{C}_{\mathbf{1}}$ ) is the correlation vector delivered by the demodulator when the symbol 0 (respectively 1) is perfectly transmitted i.e. transmitted without noise and with perfect synchronization. The equation (11) can be written in another form which shows the euclidean distance between $\mathbf{C}_{0}$ and $\mathbf{C}_{1}$. Considering the root square matrix $\mathbf{S}$ of $\mathbf{R}_{\eta}^{-1}, \mathcal{P}(\mathbf{C})$ can be expressed as:

$$
\mathcal{P}(\mathbf{C})=\frac{p_{0} e^{-\left\|\mathbf{X}-\mathbf{X}_{\mathbf{0}}\right\|^{2}}+p_{1} e^{-\left\|\mathbf{X}-\mathbf{X}_{\mathbf{0}}\right\|^{2}}}{\sqrt{\operatorname{det} 2 \pi \mathbf{R}_{\eta}}}
$$

where $\mathbf{X}=\mathbf{S} . \mathbf{C}$ and $\mathbf{X}_{0,1}=\mathbf{S} . \mathbf{C}_{0,1}$.

The equation (11) shows, by a change of variable $\widehat{\mathbf{C}}=\left(\mathbf{C}-\mathbf{C}_{\mathbf{0}}\right)$, that the mutual information $I(\mathbf{C}, b)$ will be maximized when the distance between $\mathbf{C}_{\mathbf{1}}$ and $\mathbf{C}_{\mathbf{0}}$ is maximal. With a monocycle pulse this corresponds to use for the mapping function the following time shift:

$$
\Delta=\frac{\sqrt{5-\sqrt{10}}}{\pi} \times \tau
$$

in order to maximize the performance when using two correlations.

This result deserves some comments:

- it comes in contrast with the classical choices where $\Delta$ is usually chosen larger (closer to $\tau$ ) in order to reach a greater pulses orthogonality

- such a choice results in a better robustness towards synchronization errors since it maximizes the contrast between $\mathbf{C}_{\mathbf{0}}$ and $\mathbf{C}_{\mathbf{1}}$.

This result is illustrated in the next section when studying the impact of desynchronization.

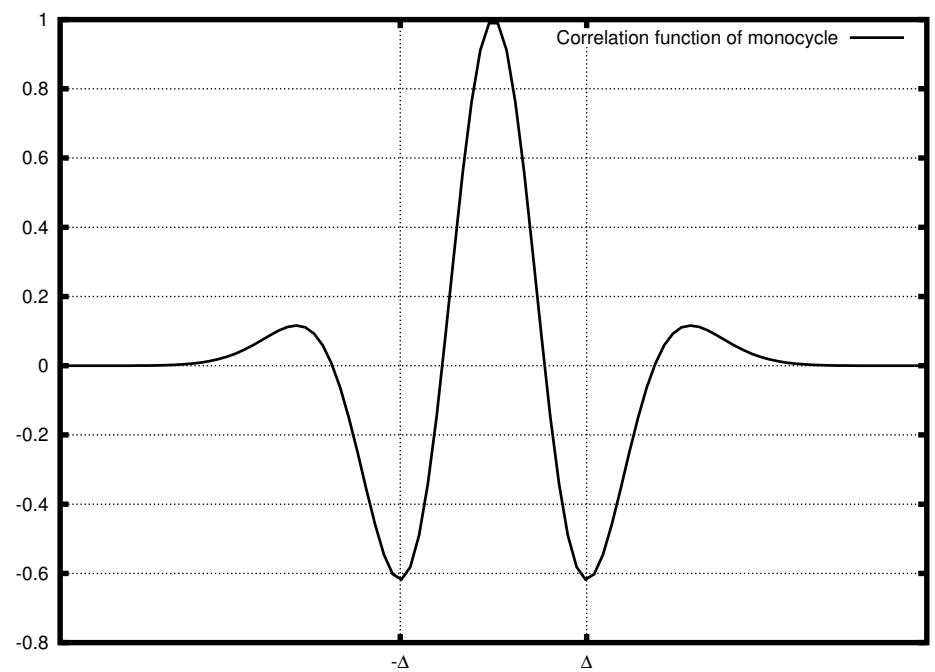

Figure 2: Correlation function of monocycle pulse

\section{B Impact of desynchronization on the link capacity}

When perfectly synchronized, the expected symbols $\mathbf{C}_{0}$ and $\mathbf{C}_{1}$ are given by

$$
\begin{aligned}
& \mathbf{C}_{0}=\left[\begin{array}{c}
S_{w}(0) \\
S_{w}(\Delta)
\end{array}\right] \\
& \mathbf{C}_{1}=\left[\begin{array}{c}
S_{w}(\Delta) \\
S_{w}(0)
\end{array}\right]
\end{aligned}
$$

The euclidean distance between these symbols is then given by: $\sqrt{2}\left(S_{w}(0)-S_{w}(\Delta)\right)$. This corresponds to a zero time offset i.e. $\delta=0$. Taking into account time desynchronization: the expected symbols are given by

$$
\begin{aligned}
\mathbf{C}_{0} & =\left[\begin{array}{c}
S_{w}(\delta) \\
S_{w}(\Delta+\delta)
\end{array}\right] \\
\mathbf{C}_{1} & =\left[\begin{array}{c}
S_{w}(\Delta-\delta) \\
S_{w}(\delta)
\end{array}\right]
\end{aligned}
$$

The time offset $\delta$ influences the link capacity by reducing the Euclidean distance between $\mathbf{C}_{0}$ and $\mathbf{C}_{1}$.

\section{Numerical results}

Figures 3 and 4 displays the link capacity versus time desynchronization $\delta$ in two configurations: single correlation demodulator (figure 3) and two correlations demodulator (figure 4).

In figure 3 we can observe an asymmetry of the capacity with respect to the sign of $\delta$. Using a single correlator demodulator is equivalent to relying on the first component of the vector $\mathbf{C}^{k}$. This explains why the effects of a negative and positive $\delta$ are different. With a two correlations demodulator figure 4 reveals that the capacity 


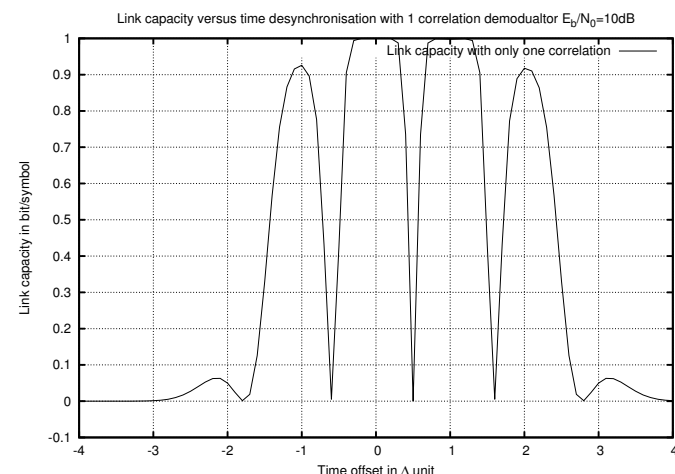

Figure 3: Link capacity versus time offset in $\Delta$ unit: single correlation demodulator

is not influenced (flat) over a relatively large range of $\delta$. Note that the capacity is maximal not only for perfect synchronization $(\delta=0)$ but also for $\delta= \pm \Delta$. $\delta=0$ corresponds to local pulses that are aligned with the received ones while $\delta= \pm \Delta$ occurs when only one local pulse is aligned with the received one (i.e. local pulse for bit 0 aligned with the received pulse corresponding to a transmitted 1 or the opposite case). In order to avoid catastrophic time offsets, this result would help in positioning an extra template for providing a correlation that would yield enough information to recover from the bad capacity values.

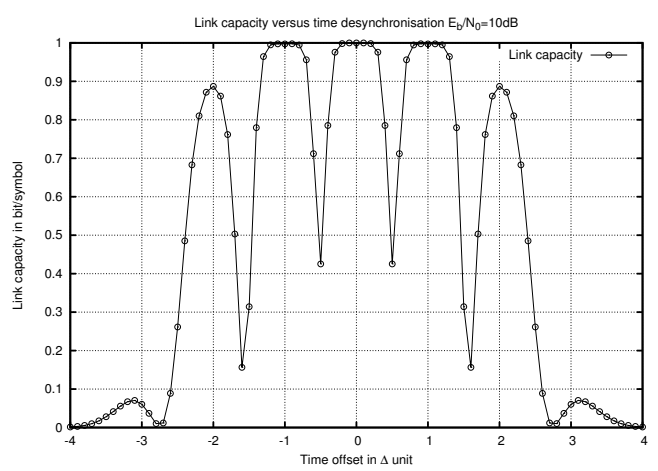

Figure 4: Link capacity versus time offset in $\Delta$ unit: two correlators demodulator

Figure 5 draws the iso-capacity curves versus signal to noise ratio and time offset (desynchronization). Each curve corresponds to equal capacity in the plane coordinates given by time desynchronization (abscissa) and bit energy to noise ratio (ordinate). As shown, a time shift around $\frac{1}{2} \Delta$ is equivalent to $7 d B$ loss in $\frac{E_{b}}{N_{0}}$. This figure can be used to study the effect of repetition (i.e. value of $N_{f}$ ) on the performance of the link. Indeed, increasing $N_{f}$ makes $\frac{E_{b}}{N_{0}}, N_{f}$ times better but the final data rate is calculated relative to $\frac{1}{N_{f} T_{f}}$ rather than $\frac{1}{T_{f}}$. Such results can be used in link adaptation algorithms:

- with perfect synchronization and without repetition the link capacity is $\frac{0.95}{T_{f}}$ at $\mathbf{5} \mathrm{dB}$ of pulse energy to noise ratio and $\frac{0.8}{T_{f}}$ at $2 \mathrm{~dB}$.

- with a repetition factor $N_{f}=2$ the link capacity is $\frac{0.95}{N_{f} T_{f}}=\frac{0.47}{T_{f}}$ at $2 \mathrm{~dB}$ of pulse energy to noise ratio and thus at $5 \mathrm{~dB}$ of bit energy to noise factor. Clearly in this case, repetition should be avoided (capacity is almost halved).

\section{A link budget for binary PPM systems with desynchroniza- tion considerations}

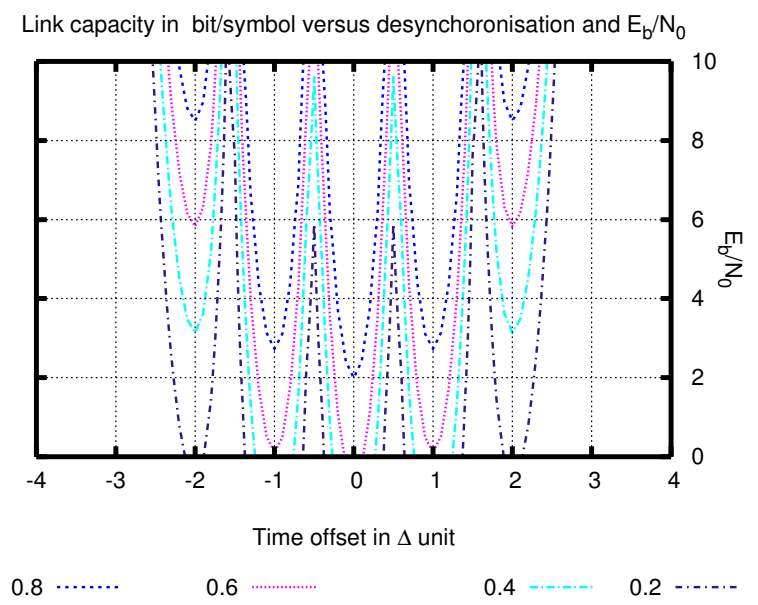

Figure 5: Iso-capacity curves versus desynchronization and $\frac{E_{b}}{N_{0}}$

In this section the previous results concerning the link capacity are exploited for evaluating the maximal data rate that can be achieved in a practical context. Two main issues are considered:

- the time jitter that can affect the local oscillator in the receiver;

- the timing uncertainties resulting from the peak detection when estimating time of arrival in the channel estimation.

Without taking account of these two effects the link budget of a binary PPM system is given by the bit energy to noise ratio

$$
\frac{E_{b}}{N_{0}}=\frac{F C C_{m a s k} \times B_{W} \times T_{f} \times P_{L}}{k_{B} T \times B_{W} \times F \times T_{p}}
$$

where

- $\mathrm{FCC}_{\text {mask }}$ is the power mask spectrum specified by the FCC: $F C C_{m a s k}=-41 \mathrm{dBm} / \mathrm{MHz}$

- $B_{W}$ is the band width of the signal. 
- $T_{f}$ is the frame time. $\frac{1}{T_{f}}$ corresponds to the pulse repetition frequency. $\frac{1}{T_{f}}=156 \mathrm{MHz}$

- $P_{L}$ is the path loss. Typically for short range we can consider:

$$
\left(P_{L}\right)_{d B}=-20 \times \log _{10}\left(\frac{4 \pi d}{\lambda}\right)
$$

- $k_{B} T$ is the classical thermal noise.

- $F$ is the noise figure of the device. For UWB systems this factor is higher than narrow band systems. It will be taken around $\mathbf{1 0 d B}$

- $T_{p}$ is the pulse length. Typically $T_{p}=4 \times \tau$

- $\lambda$ is the wave length corresponding to the center frequency of the signal.

We will consider a monocycle pulse with $\tau=200 p s$ which corresponds to a bandwidth of $\mathbf{5 G H z}$ and a center frequency of $5 \mathrm{GHz}$ thus $\lambda=0.06 \mathrm{~m}$. The time shift between 0 and 1 is given by equation eq. (13) $\Delta=86 \mathrm{ps}$.

As no repetition is assumed $N_{f}=1$, the maximum data rate can be derived from the pulse repetition frequency $\frac{1}{T_{f}}$ and the link capacity expression of the previous section.

$$
R_{\max }=\frac{\max I(C, b)}{T_{f}}
$$

Now let consider the effect of desynchronization. In order to build the complete link budget we need a model for the desynchronization that can alter the receiver. Such model is given by the oscillator time jitter and the error that affects the channel estimation.

We base our analysis using the Saleh-Valenzuella like channel models of IEE802.15.3a [5, 9]. For simplicity sake, we suppose here that the receiver detects only the first ray of the first cluster of the channel response.

A simple desynchronization model for the random variable $\delta$ can thus be written as

$$
\delta=\chi_{c}+J
$$

where

- $J$ is the time jitter resulting from the receiver oscillator phase noise. Usually $J$ is a colored Gaussian variable [7].

- $\chi_{c}$ is the error on the ray arrival time. It is supposed to be a fixed shift within packet transmission and can take values uniformally distributed in $\left[-\frac{\Lambda}{2} \frac{\Lambda}{2}\right]$ where $\Lambda$ is the average interval between two successive rays.

Figures 6 and 7 depict the iso-data rate curves versus time offset and distance between two communicating devices without or including jitter effects respectively.
Figure 6 draws the available data rate when taking into account only the error on the estimation of the first array arrival time ie $J=0$ in equation 19. Each curve corresponds to equal data rate in the plane coordinates given by distance and $\chi_{c}$.

Figure 7 shows the same result but with jitter effects included: for every value of $\chi_{c}$ the data rate is averaged over all possible values of time jitter $J$. Jitter is considered to be originated by a phase noise of $-90 \mathrm{dBc} / \mathrm{MHz}$ at $\mathbf{1 M H z}$. This is equivalent to a variance of $\mathbf{1 7 p s}$.

Comparing these two figures shows that particular points are more immune to time oscillator phase noise: typically those that corresponds to perfect estimation of ray arrival time. When perfectly synchronized the link range at $150 \mathrm{Mb} / \mathrm{s}$ is reduced from $8 \mathrm{~m}$ to $6 \mathrm{~m}$ by oscillator jitter. And with a jitterless oscillator the penalty of $\mathbf{2 5} \mathrm{ps}$ in the estimation of ray arrival time is equivalent to a loss of $4 \mathrm{~m}$ (from $8 \mathrm{~m}$ to $4 \mathrm{~m}$ ) in range. The iso-data rate curves can be helpful in system design since they allow to evaluate the optimum tradeoff between transmitting power (which influences $\frac{E_{b}}{N_{0}}$ ) and system time accuracy which depends on channel estimation and synchronization algorithms, and timing accuracy of the receiver demodulator.

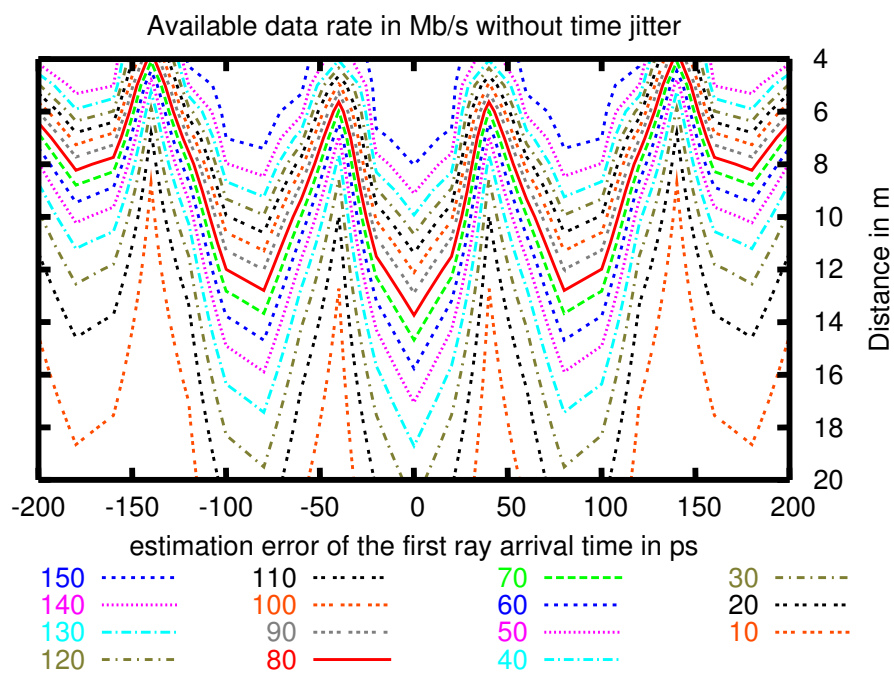

Figure 6: Data rate versus distance and desynchronization without jitter

\section{Conclusion}

In this paper we have derived the capacity of binary PPM UWB systems taking into account timing constraints considerations for a specific demodulator architecture. This evaluation provides an upper bound for a target data rate. In this study, both the time jitter caused by receiver local oscillator and also the time offset error resulting from the the channel estimation error are considered. This capacity evaluation allows to quantify the penalty in term of signal to noise ratio and also in term of link range 
Averaged available data rate in $\mathrm{Mb} / \mathrm{s}$ with jitter and desychronization

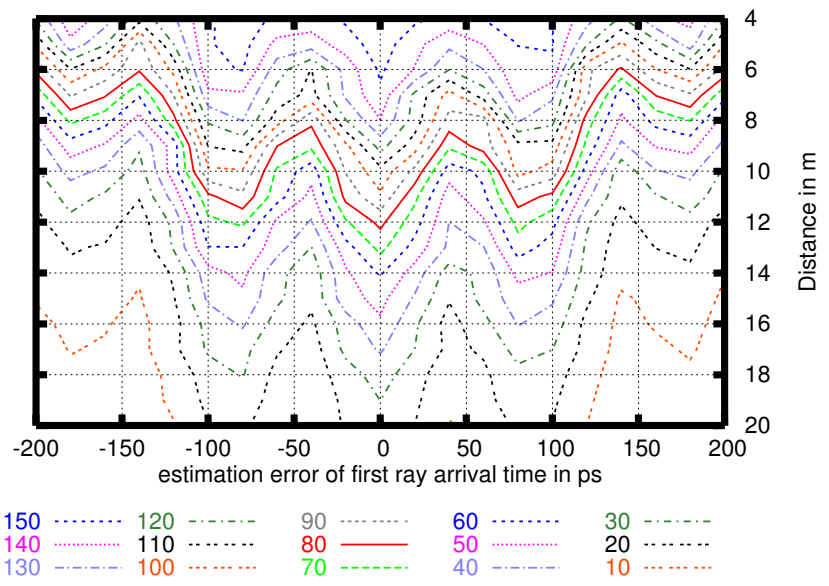

Figure 7: Data rate versus distance and desynchronization including jitter

and timing impairments of the receiver components. As shown in the last section, $\mathbf{2 m}$ can be lost in terms of coverage using typical off the shelf oscillators available on the market.

Note that by taking into account the extremum points of the link capacity, this work can be further extended to propose modifications of the demodulator architecture in order to cleverly put extra-correlations that would overcome timing constraints.

\section{References}

[1] IEEE 802.15 website. http://www.ieee802.org/15/.

[2] UCAN website. http://www.ucan.biz.

[3] Part 15 radio frequency devices. Technical report, FCC, 2003.
[4] S. Dolinar, D. Divsalar, J. Hamkins, and F. Pollara. Capacity of pulse-position modulation (ppm) on gaussian and webb channels. Technical report, 2000 .

[5] Jeff Foerster. Channel modeling sub-committe report final. Technical report, IEEE, 2002.

[6] C.J. Le Martret and Georgios B. Giannakis. Alldigital impulse radio with multiuser detection for wireless cellular systems. IEEE Transactions on Communications, September 2002.

[7] Rick Poore. Phase noise and jitter. Technical report, Agilent, 2001.

[8] J. G. Proakis. Digital Communications. Mc Graw Hill, New York, USA, 3rd ed., 1995.

[9] A.M. Saleh and A.A. Valenzuela. A statistical model for indoor multipath propagation. IEEE Journal on Selected Areas in Communications, pages 128-137, February 1987.

[10] R Schreier. ADCs and DACs: Marching towards the antenna. In ISSCC GigaHertz-Radio Front Ends Workshop, 2003.

[11] Robert H. Walden. Analog-to-digital converter survey and analysis. IEEE Journal on Selected Areas in Communications, 1999.

[12] M.Z. Win and R.A. Scholtz. Impulse radio: How it works. IEEE Communication Letters, 2:36-38, February 1998.

[13] L. Zhao and A. M. Haimovich. Capacity of mary ppm ultra-wideband communications over awgn channels. In Porceeding of VTC Fall conference, 2001. 